AMANDA BARON CAMPAÑA

\title{
A intolerância à glicose que se instala precocemente em ratos retarda o desenvolvimento puberal e desregula o metabolismo lipídico e glicídico
}

Dissertação de Mestrado

apresentada ao Instituto de Ciências

Biomédicas da Universidade de São Paulo, para a obtenção do Título de Mestre em Ciências (Fisiologia Humana).

Área de Concentração: Fisiologia Humana

Orientador: Fábio Bessa Lima 


\section{RESUMO}

CAMPAÑA, A.B. A intolerância à glicose que se inicia precocemente em ratos retarda o desenvolvimento puberal e desregula o metabolismo lipídico e glicídico, 2008. Tese de Mestrado (Fisiologia Humana) - Instituto de Ciências Biomédicas, Universidade de São Paulo, São Paulo, 2007.

A crescente prevalência de diabetes mellitus (DM) e sua disseminação mundial caracterizam esta moléstia como um importante problema de saúde pública. Além disso, nos últimos anos, tem-se observado uma incidência cada vez maior de diabetes tipo 1 e 2 em crianças e adolescentes, justificando, portanto, a importância de trabalhos que esclareçam as especificidades do DM neste período específico do desenvolvimento. Somado a isto, estudos prévios desenvolvidos em nosso laboratório encontraram uma piora do quadro metabólico em ratos diabéticos, em modelo de indução por estreptozotocina no período neonatal. Frente a isto, este trabalho teve por objetivo caracterizar o metabolismo de glicose em tecido adiposo branco de ratos no período da puberdade, avaliando-se como a resistência à insulina deste período se manifesta nos adipócitos de ratos normais e intolerantes à glicose. Ratos Wistar machos foram divididos em dois grupos experimentais no quinto dia de vida: o grupo controle (CO), tratados apenas com o veículo (tampão citrato) e o grupo estreptozotocina (STZ), que recebeu injeção intraperitoneal (120mg/kg p.c.) de droga. Parâmetros de desenvolvimento corporal e maturação sexual foram estudados entre a $5^{a}$ e a $10^{a}$ semanas de vida dos animais, a fim de se identificar a fase inicial da puberdade. Este acompanhamento demonstrou a $6^{a}$ e $7^{a}$ semanas como importante fase da puberdade, onde se inicia o estirão de crescimento e desenvolvimento sexual. As dosagens hormonais de insulina e glicemia, somadas ao teste oral de tolerância à glicose revelaram uma franca resistência à insulina no período da puberdade, a qual produziu prejuízos no metabolismo de glicose em adipócitos isolados. A injeção de estreptozotocina no quinto dia de vida produziu um quadro de intolerância à glicose em nossos animais. Esta alteração no controle glicêmico, obtida pela injeção da droga diabetogência, resultou em um atraso de aproximadamente uma semana no início do estirão de crescimento e desenvolvimento sexual no grupo STZ. Neste grupo, as respostas metabólicas estimuladas por insulina em adipócitos 
isolados apresentaram-se normais ou até mesmo mais intensas no período inicial da puberdade, com piora ao longo do desenvolvimento, atingindo níveis mais baixos do que os observados em animais controle. Enquanto nos animais normais, a resistência fisiológica à insulina observada tendeu a se abrandar ao longo do desenvolvimento. Nos animais CO, o fenômeno de resistência fisiológica à insulina na puberdade é transitório ao passo que em animais STZ intolerantes à glicose, o quadro de resistência é insidioso e se agrava com o passar do tempo, tendo, portanto, um caráter diferente que não condiz com o que seria uma resistência fisiológica. Assim, os mecanismos envolvidos neste processo em ratos com intolerância à glicose ou, mesmo, francamente diabéticos seriam de natureza diferente e o desenvolvimento e a progressão dos processos patológicos envolvidos merecem uma investigação mais aprofundada.

Palavras-chave: Intolerância à glicose. Desenvolvimento genital puberal. Tecido adiposo branco. Resistência à insulina. Metabolismo de glicose. Lipogênese. Lipólise. 


\begin{abstract}
CAMPAÑA, A.B. The glucose intolerance that is manifested early in the life in rats retards pubertal development and deranges carbohydrate and lipid metabolism, 2008. Master Thesis (Human Physiology) - Instituto de Ciências Biomédicas, Universidade de São Paulo, São Paulo, 2007.
\end{abstract}

The growth in the prevalence of diabetes mellitus (DM) and its world spreading characterizes this disease as an important public health problem. Besides, in the last years, it has been observed a larger incidence of type 1 and $2 \mathrm{DM}$ in children and adolescents, justifying, therefore, the need for more researches to understand the DM peculiarities of this specific period of development. In addition, previous studies developed in our laboratory described a worsening of the metabolic picture in diabetic rats during puberty, in a model of neonatally streptozotocin-induced DM. In face of this, the present work aimed to characterize the carbohydrate metabolism of white adipose tissue from neonatally streptozotocin-injected rats in the period of puberty in order to assess the possible influence of the physiological insulin resistance characteristic of puberty on the course of the diabetic status. Five-day old male Wistar rats were divided into two experimental groups which were or not injected intraperitoneally with streptozotocin (120 mg/kg b.w.): the control group (CO), just treated with the vehicle (citrate buffer) and the streptozotocin group (STZ), injected with the drug. Body development and sexual maturation were weekly evaluated between the $5^{\text {th }}$ and $10^{\text {th }}$ weeks of life, in order to identify early and late phases of the puberty. This protocol showed that the period comprised by the 6th and 7th weeks is an important early phase of the puberty, characterized by the growth spurt of somatic and sexual development. Through the fasting blood glucose and insulin determinations and the response to an oral glucose tolerance test done at the $6^{\text {th }}$ and $7^{\text {th }}$ weeks of life both the insulin resistance and a light degree of glucose intolerance were determined in nondiabetic rats. On the other hand, the streptozotocin-injected (STZ) rats in the fifth day of life showed very intense glucose intolerance despite the fact that their fasting blood glucose were slightly above that of control animals. This metabolic disturbance resulted in approximately one week delay of in the beginning of the pubertal growth and sexual development. In this group, the 
insulin-stimulated metabolic responses in isolated adipocytes appeared normal or even more intense than in $\mathrm{CO}$ rats in the early puberty, and were progressively worsening, reaching a very poor response in late puberty and early adult life. In the $\mathrm{CO}$ group, on the contrary, the physiologic insulin resistance detected in early puberty vanished during the course of puberty and was completely recovered at the early adulthood. In the animals $\mathrm{CO}$, the phenomenon of physiologic insulin resistance in the puberty is transient while, in glucose-intolerant STZ animals, the resistance starts insidiously and worsens in the course of puberty, suggesting that the developing insulin resistance in STZ-treated rats during puberty has a different characteristic of that seen in normal rats and, therefore, the mechanisms underlying this process is of a different nature, requiring a more detailed and deeper investigation.

Key words: Glucose intolerance, Pubertal development, White adipose tissue, Insulin resistance, Glucose metabolism, Lipogenesis, Lipolysis. 


\section{INTRODUÇÃO}

O Diabetes Mellitus (DM), doença metabólica resultante de uma deficiência na secreção e/ou na ação da insulina, é, atualmente, um problema de saúde pública, exigindo importantes gastos com seu acompanhamento e tratamento, bem como com as complicações produzidas pela hiperglicemia crônica. Esta doença contribui para o aumento da predisposição ao desenvolvimento de outras morbidades como as macro e micro-angiopatias (cardíacas, neurais, oftálmicas e renais), além de outros comprometimentos sistêmicos diversos levando ao aumento na taxa de mortalidade prematura (ANNIS et al., 2005).

A sua expressiva disseminação pelo mundo tem ocorrido tão rapidamente que esta doença passou a ser classificada como epidemia global (OGLESBY et al., 2006). Sua prevalência cresceu significativamente durante as últimas décadas e a expectativa é de que este crescimento continue nos próximos anos (CONNOR et al., 2006).

Este importante problema de saúde pública mundial consiste em um conjunto de desordens no metabolismo de glicose, proteínas e gorduras resultantes de um efeito ineficaz da insulina (secreção e/ou ação deficiente) em seus tecidos-alvo.

A insulina, hormônio produzido pelas células $\beta$ do pâncreas, tem por função, juntamente com o glucagon, coordenar o fluxo e destino metabólico da glicose endógena, dos ácidos graxos livres, aminoácidos e de outros substratos que garantem o suprimento das necessidades energéticas durante o estado basal e no exercício. Além disso, coordenam a distribuição eficiente dos nutrientes adquiridos durante as refeições (GENUTH, 2004). Neste quadro de regulação do metabolismo, a insulina exerce um papel anabólico, estimulando a captação de glicose em suas células-alvo, assim como processos de armazenamento de energia (lipogênese e síntese de glicogênio e proteínas) e inibe processos catabólicos nestes mesmos tecidos, tal como lipólise, glicogenólise e degradação de proteínas.

A insulina é secretada pelas células $\beta$, principalmente, em resposta a um aumento dos níveis de glicose plasmática. Além da glicose, outros estímulos 
podem produzir a secreção de insulina: aminoácidos provenientes da digestão de proteínas da refeição, principalmente lisina e arginina, hormônios gastrintestinais (polipeptídeo inibidor gástrico (GIP) e peptídeo 1 semelhante ao glucagon (GLP-1)) liberados em resposta às refeições e outros hormônios, dentre os quais, cortisol e hormônio de crescimento (antagonistas da insulina).

Os principais tecidos alvos da insulina são: músculo, tecido adiposo e fígado.

No músculo, a insulina estimula a captação de glicose pelas células e este substrato pode sofrer oxidação ou ser armazenado na forma de glicogênio. Este hormônio estimula também enzimas envolvidas nestes dois processos: a piruvato desidrogenase (oxidação) e a glicogênio sintetase (síntese de glicogênio). Além disso, a insulina age aumentando a captação de aminoácidos e a síntese de proteínas. Sua ação inibitória neste tecido se dá sobre a glicogenólise e sobre a degradação de proteínas e do triacilglicerol dos depósitos musculares.

No tecido adiposo, este hormônio anabólico estimula a lipogênese através do aumento da captação de glicose e da disponibilidade de ácidos graxos livres (AGLs). A glicose oferece substrato para a síntese de triacilgliceróis pelo tecido adiposo, podendo ser convertida, no interior do adipócito, em a-glicerofosfato, utilizado na esterificação de ácidos graxos, ou diretamente em ácidos graxos, o que ocorre em menor grau.

O aumento da disponibilidade de AGLs é obtido pela indução da lipase de lipoproteína que catalisa a hidrólise dos triglicerídeos das lipoproteínas de densidade muito baixa (VLDL) e dos quilomícrons. Neste tecido, a insulina tem efeito inibitório sobre a lipólise através da inibição da lipase hormônio-sensível.

No fígado, a insulina tem efeito estimulatório sobre a captação de glicose pela indução da glicoquinase hepática (fosforilação de glicose), o que diminui a concentração de glicose no interior do hepatócito e facilita sua entrada através do GLUT-2, devido à alteração do gradiente de concentração entre o meio externo e o hepatócito. A glicose é, então, armazenada na forma de glicogênio, o que também é estimulado pela insulina através da ativação do complexo enzimático da glicogênio sintetase. A insulina possui, ainda, efeito 
anticetogênico e lipogênico no fígado, além do efeito inibitório sobre a gliconeogênese e glicogenólise.

O conjunto de ações da insulina nos diferentes tecidos-alvo produz um importante controle da glicemia, impedindo um aumento exacerbado dos níveis glicêmicos frente à ingestão de carboidratos. Sua ação, somada a dos hormônios contra-reguladores, mantém a concentração de glicose no sangue em níveis normais.

O DM compõe um grupo clínica e etiologicamente heterogêneo de desordens metabólicas que possuem a hiperglicemia como característica comum (THE EXPERT COMMITTEE ON THE DIAGNOSIS AND CLASSIFICATION OF DIABETES MELLITUS, report 2003). Desta forma, esta moléstia pode ser classificada em três tipos principais: diabetes mellitus tipo1 (T1DM), diabetes mellitus tipo 2 (T2DM) e diabetes mellitus gestacional (GDM).

O T1DM se caracteriza por uma secreção deficiente de insulina em conseqüência de uma destruição das células $\beta$ do pâncreas (95\% dos casos devido a processos auto-imunes; 5\% idiopáticos), variando desde uma secreção parcial até uma ausência total deste hormônio. Na ausência da insulina, os três tecidos-alvos principais não apenas deixam de captar adequadamente os nutrientes absorvidos, como também continuam a liberar glicose, aminoácidos e ácidos graxos para a corrente sanguínea a partir de seus depósitos de armazenamento (MASHARANI et al., 2006).

No T2DM, a principal alteração encontrada é um prejuízo da ação da insulina em conseqüência da resistência a este hormônio. Sua secreção pode estar normal, parcialmente deficiente ou mesmo elevada, embora funcionalmente insuficiente.

O GDM é uma intolerância a carboidratos com início ou primeiro diagnóstico durante a gravidez. Este diagnóstico é independente do uso de insulina ou da persistência desta condição após o parto e não se aplica a mulheres com diagnósticos prévios de diabetes (JOVANOVIC et al., 2001).

Existem, ainda, outros tipos de diabetes secundários a alterações específicas, tais como, defeitos genéticos nas células $\beta$, defeitos genéticos causadores de anormalidades na ação da insulina, doenças do pâncreas exócrino, endocrinopatias (ex: excesso de hormônios antagonistas de insulina), drogas que prejudicam a ação da insulina e infecções. 
Apesar do diabetes ser uma doença metabólica de etiologia heterogênea, seus diferentes tipos são caracterizados por hiperglicemia de jejum ou elevados níveis de glicose plasmática durante um teste oral de tolerância à glicose (OGTT) (THE EXPERT COMMITTEE ON THE DIAGNOSIS AND CLASSIFICATION OF DIABETES MELLITUS, 2003).

O T1DM, segundo dados da American Diabetes Association (2004), atinge $5-10 \%$ dos pacientes diabéticos, enquanto o T2DM atinge $90-95 \%$ dos portadores da moléstia.

O T1DM ocorre em pessoas com predisposição genética como conseqüência de uma destruição auto-imune das células $\beta$ das ilhotas pancreáticas (SKYLER et al., 2002) com contribuição em maior ou menor grau de fatores ambientais. Os fatores ambientais possivelmente iniciam ou disparam o processo que leva à destruição das células $\beta$ e ao início do diabetes (ONKAMO, 1999).

A incidência do T1DM parece ter aumentado em quase todas as regiões do mundo com crescimento rápido em regiões específicas, apresentando um alto grau de variabilidade de incidência entre as diferentes etnias e uma tendência a um início cada vez mais precoce (ATKINSON et al., 2001; EURODIAB, 2000; HAYNES, 2004; ONKAMO, 1999;). Onkamo (1999), a partir de uma revisão bibliográfica sobre o panorama mundial de T1DM, concluiu que a incidência desta moléstia será 40\% maior em 2010 do que os valores observados em 1998. Ainda segundo este trabalho, a incidência global de T1DM tem aumentado no grupo de faixa etária entre 0-14 anos. Além disso, um estudo realizado por pesquisadores europeus em que se examinou este mesmo aspecto do T1DM em função da idade, demonstrou um aumento da incidência em 6,3\% em crianças com idade entre $0-4$ anos, 3,1\% entre aquelas com 5-9 anos e 2,4\% na faixa etária de 10-14 anos.

Este mesmo fenômeno tem sido observado em relação aos casos de T2DM. Pediatras, diabetologistas e outros profissionais de saúde têm reconhecido a emergência de uma epidemia de T2DM em jovens nos últimos 20 anos, afetando primariamente minorias étnicas (ROSENBLOOM et al., 1999). O T2DM é uma importante causa de morbidade e mortalidade entre adultos e tem sido diagnosticada cada vez mais freqüentemente em crianças e adolescentes (CALLAHAN et al., 2000). A idade média em que se realizam os 
diagnósticos de T2DM em crianças americanas é entre os 12 e 14 anos, período correspondente à puberdade.

A crescente prevalência de T2DM e obesidade em crianças é um sintoma do efeito da globalização e industrialização que afeta todas as sociedades com o sedentarismo e má alimentação, importantes fatores envolvidos na gênese destas doenças (ZIMMET, 2001). Obesidade e ganho de peso são os maiores fatores de risco para o desenvolvimento do diabetes (MOKDAD, 2001). O risco de diabetes tem sido relatado como 2 vezes maior em pessoas com obesidade branda, 5 vezes maior naqueles com obesidade moderada e 10 vezes maior em obesos severos (PI-SUNYER, 1993).

Apesar da experiência e conhecimento acerca da epidemiologia, fisiopatologia e aspectos clínicos do T2DM em adultos, sabe-se pouco sobre esta doença em crianças (AMERICAN DIABETES ASSOCIATION, 2000).

Assim, frente ao cenário mundial do DM descrito acima, somado à sua emergência em crianças e adolescentes, acreditou-se ser de grande contribuição um estudo sobre o metabolismo de glicose em animais com diabetes mellitus neste período específico do desenvolvimento e suas repercussões sobre esta moléstia. Para tanto, foi utilizado um modelo de diabetes mellitus induzido por estreptozotocina (STZ) em período neonatal.

A ação diabetogênica da STZ, assim como a evolução das alterações produzidas nas células do pâncreas foi, primeiramente, descrita por Rakieten, Rakieten e Nadkarni em 1967 (JUNOD et al., 1969).

A STZ [2-desoxi-2-(3-metil-3-nitrosoureido)-D-glicopiranose] é um antibiótico de amplo espectro com ações oncolítica e oncogênica, com propriedades diabetogênicas. A ação diabetogênica é mediada por uma destruição seletiva das células $\beta$ do pâncreas e tem sido largamente utilizada como um método de indução de DM em animais experimentais, além de ser empregada no tratamento de tumores malignos das células $\beta$ e outros neoplasmas em humanos (ROSSINI et al., 1977).

Os mecanismos celulares exatos da ação tóxica da STZ em sua atividade diabetogênica ainda não estão completamente elucidados, mas muitos estudos têm esclarecido alguns componentes desta ação.

A sua ação tóxica sobre as células $\beta$ das ilhotas pancreáticas tem um componente auto-imune. Múltiplas doses subdiabetogênicas de STZ produzem 
lento e progressivo aumento na glicose plasmática em camundongos dentro de 5-6 dias após as injeções, em associação com pronunciada insulite e indução de vírus tipo $\mathrm{C}$ dentro das células $\beta$ (ROSSINI et al., 1977).

O envolvimento de processos auto-imunes na sua ação tóxica sobre as células $\beta$ também foi demonstrado em camundongos athymic nude (nu/nu) e euthymic nude (+/nu) tratados com múltiplas doses subdiabetogênicas de STZ (PAIK et al.,1980). Os efeitos tóxicos só foram observados em camundongos que possuíam o timo preservado. A restauração da ação do timo, através de um transplante desta glândula para os camundongos nu/nu, recuperou a suscetibilidade destes animais às ações tóxicas das múltiplas doses de STZ, evidenciando o envolvimento de processos imunes na ação tóxica desta droga.

Alterações relacionadas aos transportadores de glicose (GLUTs) também têm sido descritas em modelos de diabetes induzido por STZ. Ratos tratados com a droga apresentaram uma diminuição do conteúdo protéico de GLUT-4, enquanto houve um aumento de sua fosforilação, resultante de uma diminuição da capacidade da insulina em estimular a desfosforilação de GLUT-4 neste modelo de diabetes (BEGUM et al., 1992). Estas alterações foram acompanhadas de concomitante redução do transporte de glicose estimulado por insulina e de uma falha desta em estimular a translocação de GLUT-4 (BEGUM et al., 1992; KARNIELI et al., 1981).

A STZ, quando administrada por via intraperitoneal, produz uma redução dose-dependente de nicotinamida adenina dinucleotídeo (NAD) nas ilhotas pancreáticas, a qual é proporcional ao grau de diabetogenicidade. Tem sido demonstrado que a STZ inibe a incorporação de nicotinamida em NAD nas ilhotas pancreáticas de ratos. Esta diminuição de NAD é acompanhada por subseqüente necrose das células $\beta$ do pâncreas (ANDERSON et al., 1974).

A ação da STZ sobre o aumento de glicose plasmática e a alteração na secreção de insulina está bem estabelecida, apesar dos mecanismos de toxicidade da droga não estarem completamente elucidados. Além destas alterações, este modelo de diabetes produz também resistência à insulina (BLONDEL, 1990; KERGOAT, 1985; SERRADAS, 1993; WEIR, 1981).

Junod et al. (1969), afirmam, em seus estudos, haver uma relação entre a dose de STZ e intensidade das alterações metabólicas produzidas, confirmando a ação altamente específica da STZ sobre as células $\beta$ do 
pâncreas e a sua possível utilização para a obtenção de quadros de diabetes em diferentes graus de severidade.

Takada (2003), estudando o perfil metabólico de animais (ratos) portadores do DM induzido no período neonatal ( $5^{\circ}$ dia de vida) por injeção intraperitoneal de STZ (150 mg/kg de p.c.), constatou que esses animais na fase adulta (12 semanas de vida) apresentavam um quadro clínico característico do DM (hiperglicemia de jejum, hiperfagia, polidipsia, glicosúria e poliúria), com reservas reduzidas de insulina pancreática, porém com forte componente de resistência periférica a este hormônio. Neste estudo, os ratos tratados com STZ apresentaram ligeira hiperglicemia após o desmame, com um agravamento acentuado do quadro glicêmico entre a $6^{a}-8^{a}$ semana de vida. Neste período, correspondente à fase de puberdade do animal, a existência de uma resistência à insulina fisiológica é bem conhecida. Assim, como o agravamento do quadro diabético persistiu até a idade adulta, é possível que fatores desencadeados na puberdade tenham participado na gênese da resistência. Frente a isso, um estudo mais detalhado deste período específico neste modelo animal contribuirá para uma maior compreensão sobre os aspectos da relação entre puberdade e as alterações do metabolismo de glicose características do diabetes.

Além disso, a relação do metabolismo de glicose em adipócitos isolados com o desenvolvimento e a maturação sexual normal de ratos, também é de grande importância para uma melhor compreensão sobre os efeitos da puberdade no metabolismo energético destes animais. Permitindo, assim, um aprofundamento nas especificidades do metabolismo destes animais largamente empregados em pesquisas científicas.

Somado a isso, está a importância do tecido adiposo. Este tecido é um órgão metabólico e endócrino altamente complexo, ativo e essencial. Ele responde não somente aos sistemas hormonais tradicionais e ao sistema nervoso central, como também expressa e secreta fatores com importantes funções endócrinas. Assim, este trabalho visa contribuir também elucidando características específicas do metabolismo deste importante tecido no período da puberdade.

A respeito da puberdade em ratos sabe-se que a capacidade reprodutiva é atingida por volta do $50^{\circ}$ dia de vida ( $7^{a}$ semana) quando se inicia a 
espermatogênese nos testículos. A espermatogênese é produzida por um aumento dos níveis de gonadotrofina na circulação e nos níveis de andrógeno testicular nos ratos adultos (FOSTER et al., 1985; KETELSLEGERS et al., 1978; OJEDA et al., 1984; PAYNE et al., 1977).

O principal evento da puberdade é o início da secreção pulsátil de hormônio liberador de gonadotropina $(\mathrm{GnRH})$. Esse novo padrão de secreção de GnRH comanda uma secreção pulsátil de hormônio folículo estimulante (FSH) e de hormônio luteinizante (LH) pelo lobo anterior da hipófise. A secreção pulsátil de FSH e LH estimula a secreção de hormônios esteróides pelas gônadas, produzindo um aumento dos níveis circulantes destes hormônios (COSTANZO, 2004).

Estes pulsos de secreção hormonal, resultantes do amadurecimento do eixo hipotálamo-hipofisário-gonadal, controlam funções que visam à capacitação do indivíduo para a reprodução. Em machos, estes novos padrões de secreção hormonal ( $\mathrm{GnRH}$, LH, FSH) produzem um aumento da produção e liberação de testosterona pelas células de Leydig, estimuladas pelo LH, e o início da diferenciação das células germinativas em espermatozóides, controlada por FSH e testosterona.

Outro importante evento desencadeado na puberdade é o aumento da amplitude do pulso de secreção do hormônio de crescimento $(\mathrm{GH})$, o que é estimulado pela ação da testosterona e estradiol na hipófise. O GH é necessário para o crescimento linear normal e também afeta muitos aspectos do metabolismo corporal, sendo classificado como anabólico, lipolítico e diabetogênico (THORNER et al., 1988). Ele age em sinergia com a testosterona promovendo o aumento de massa muscular. Além disso, o IGF-1 e o GH potencializam o desenvolvimento gonadal e a secreção dos hormônios esteroidais sexuais (GENUTH, 2004).

O aumento dos níveis de esteróides sexuais no período da puberdade é acompanhado de alterações metabólicas como a presença de resistência à insulina e modificações na secreção de leptina. Estas transformações características da puberdade podem influenciar o quadro de diabetes induzido por estreptozotocina.

Há uma rica comprovação experimental de que insulina e esteróides gonadais interagem sobre os tecidos. Em concentrações fisiológicas, 
testosterona e estradiol estão envolvidos com a manutenção da sensibilidade normal à insulina. Entretanto, fora dos níveis fisiológicos, estes esteróides podem promover resistência à insulina. A elevação das concentrações de esteróides sexuais característica do período de puberdade está associada a uma diminuição da sensibilidade à insulina e seus efeitos parecem estar restritos ao metabolismo de glicose nos tecidos periféricos (LIVINGSTONE e COLLISON, 2002).

A resistência à insulina na puberdade tem sido descrita em diversos estudos e a diminuição da sensibilidade à insulina está associada a um aumento de sua secreção. Porém, o propósito destas modificações sobre a ação e secreção da insulina neste período não está esclarecido (GORAN et al., 2001). Em todos os casos de resistência à insulina há uma resposta biológica diminuída em seus tecidos-alvo. A resistência à insulina causa, inicialmente, uma diminuição das taxas de clareamento (clearance) sangüíneo da glicose, predominantemente para músculos esqueléticos e, em menor extensão, para o tecido adiposo. Por esta razão, desenvolve-se uma tendência à hiperglicemia. Em uma tentativa para reajustar este desequilíbrio glicêmico, uma maior quantidade de insulina é secretada pelas células $\beta$ do pâncreas, resultando em hiperinsulinemia (LIVINGSTONE et al., 2002).

As alterações dos padrões de secreção de leptina características do período da puberdade também podem estar relacionadas com uma possível piora do quadro de DM induzido por STZ.

A leptina é um hormônio produzido pelas células adiposas e o aumento de seus níveis tem sido proposto como um fator retro-alimentador negativo para o cérebro, produzindo decréscimo da ingestão alimentar, aumento do gasto de energia e resistência à obesidade. Somada a esta função, que tem sido proposta como primária, a leptina circulante também parece desempenhar um importante papel sobre o eixo neuroendócrino, incluindo a regulação da reprodução. Confirmando estas hipóteses, o rato ob/ob (com total deficiência de leptina) apresenta infertilidade e hipogonadismo, somada à hiperfagia e à obesidade mórbida. $O$ tratamento crônico com leptina não reduz apenas a ingestão alimentar e o peso, mas também restabelece a puberdade e a fertilidade (AHIMA et al., 1997). Uma mutação no gene que codifica a leptina e o seu receptor resultam em uma falha no início da puberdade e no 
desenvolvimento das características sexuais secundárias (CLÉMENT et al., 1998; STROBEL et al., 1998), indicando sua importância para este processo de maturação sexual.

Mantzoros et al. (1997) sugerem em seu estudo que há um breve pulso de leptina precedendo o desenvolvimento da puberdade no sexo masculino. Outros estudos revelam um dimorfismo nas taxas de leptina, com um aumento de sua secreção na puberdade em meninas e uma diminuição em meninos. Existem evidências em humanos e em roedores de que testosterona exerce uma regulação negativa sobre a leptina, enquanto o estrógeno age como um regulador positivo (CASABIELL, 2001) Estas diferenças são explicadas em parte devido às diferenças de índice de massa corpórea e níveis hormonais (AHMED et al., 1999).

Leptina induz a secreção de IL-1 $\beta$ em cultura de ilhotas pancreáticas humanas, enquanto diminui a expressão de IL-1Ra (antagonista do receptor de IL-1) nas células $\beta$. IL-1 $\beta$ produz uma diminuição da função das células $\beta$ e apoptose nas ilhotas pancreáticas humanas. O IL-1Ra é um antagonista natural de IL-1 $\beta$ e protege a cultura de ilhotas humanas de sua ação tóxica (MAEDLER et al., 2004). Assim, um aumento da secreção de leptina produziria um aumento da secreção de IL-1 $\beta$ com conseqüente prejuízo da atividade das células $\beta$ do pâncreas. Além disso, Cohen, Novick e Rubinstein (1996) concluíram em seus estudos que a exposição de células pancreáticas a concentrações de leptina semelhantes às encontradas em indivíduos obesos causa diminuição de diversas ações induzidas pela insulina, incluindo a fosforilação em tirosina do substrato 1 do receptor de insulina (IRS-1), ligação da molécula da proteína 2 ligante ao receptor de fator de crescimento (GRB2) ao IRS-1 e infra-regulação (down-regulation) da gliconeogênese.

No âmbito das ações periféricas da insulina, a leptina prejudica tanto o estímulo à utilização de glicose e ao processo de lipogênese quanto à inibição da lipólise em adipócitos isolados. No músculo esquelético, a presença de leptina promove o aumento da captação de glicose, da síntese de glicogênio e da oxidação de ácidos graxos. Por outro lado, a insulina aumenta a expressão e a liberação de leptina em adipócitos e em fragmentos de gordura periepididimal incubados com o hormônio pancreático (BRITO, 2005). 
Os estudos sobre a relação entre leptinemia e ação da insulina divergem a respeito dos efeitos da primeira sobre a última, porém em todos eles a importância desta relação entre leptinemia e ação insulínica está estabelecida. Assim, as alterações no padrão de secreção da leptina ocorridas na puberdade influenciam as atividades insulino-estimuladas, justificando-se sua importância para esta pesquisa.

As especificidades do período da puberdade com suas alterações hormonais características, somada ao panorama mundial do DM, apresentando uma emergência do subtipo 1 e 2 em crianças e adolescentes, e às observações do estudo de Takada (2003) levam a conclusão sobre a importância de se caracterizar o metabolismo celular de glicose neste período específico nos ratos tratados com STZ. Além disso, a importância endócrina do tecido adiposo e as especificidades hormonais do período da puberdade direcionaram um estudo sobre a relação do metabolismo de glicose neste tecido e a puberdade. Esta pesquisa visa, portanto, uma maior compreensão sobre os aspectos da relação entre puberdade e sua possível influência sobre o metabolismo de glicose no tecido adiposo branco de animais controle e daqueles em que foi injetada STZ. 


\section{CONCLUSÕES}

6.1 Existe resistência fisiológica à insulina no período da puberdade em ratos, com glicemia e insulinemia de jejum aumentadas, e com a presença de intolerância à glicose.

6.2 Os altos níveis de testosterona e GH e IGF-1 característicos da puberdade parecem desempenhar um importante papel no desenvolvimento da resistência fisiológica à insulina em ratos no período da puberdade.

6.3 O período entre a $6^{\mathrm{a}}$ e $7^{\mathrm{a}}$ semanas de vida consiste em uma importante fase da puberdade em ratos, quando ocorre o estirão de crescimento e desenvolvimento sexual dos animais.

6.4 A intolerância à glicose no período puberal produziu prejuízos no metabolismo deste substrato energético apenas no coxim adiposo SC, indicando possível relação entre o tecido adiposo PE e o desenvolvimento do sistema reprodutor.

6.5 A intolerância à glicose produzida pela injeção intraperitoneal de esptreptozotocina produziu atraso no desenvolvimento e maturação sexual de ratos em aproximadamente uma semana, apresentando prejuízos persistentes no desenvolvimento em algumas das estruturas estudadas.

6.6 O metabolismo de glicose apresentou deficiência na resposta ao estímulo por insulina nos adipócitos isolados dos coxins SC e PE, devido ao quadro de intolerância produzida pela estreptozotocina.

6.7 O período da puberdade nos animais do grupo STZ apresentou uma melhora da resposta dos adipócitos ao estímulo de insulina devido a prováveis atuações de mecanismos compensatórios frente à importância deste tecido para este período característico do desenvolvimento. 
6.8 O quadro de resistência à insulina dos animais intolerantes à glicose, ou mesmo francamente diabéticos é de natureza diferente à resistência fisiológica à insulina do período da puberdade. 


\section{REFERÊNCIAS}

AGBAJE, I.M. et al. Insulin dependent diabetes mellitus: implications for male reproductive function. Human Reproduction, v. 22, n.7, p. 1871-1877, 2007.

AHIMA, R.S.; DUSHAY, J.;FLIER, S.N.; PRABAKARAN, D.; FLIER, J.S. Leptin accelerates the onset of puberty in normal female mice. Journal of Clinical Investigation, v. 99, n. 3, p. 391-395, 1997.

AHMED, M.L.; ONG, K.K.L.; COX, L.; DRAYER, N.; PERRY, L.; PREECE, M.A.; DUNGER, D.B. Longitudinal Study of Leptin Concentrations during Puberty: Sex Differences and Relationship to Changes in Body Composition. The Journal of Clinical Endocrinology \& Metabolism, v. 84, n.3, p. 899-905, 1999.

AMERICAN DIABETES ASSOCIATION. Type 2 Diabetes in Children and Adolescents. Diabetes Care, v. 23 n. 3, p. 381-389, 2000.

AMERICAN DIABETES ASSOCIATION. Diagnosis and classification of diabetes mellitus. Diabetes Care, 2004.

AMIEL, S.A.; CAPRIO, S,; SHERWIN, R.S.; PLEWE, G.; HAYMOND, M.W.; TAMBORLANE, W.V. Insulin resistance of puberty: a defect restricted to peripheral glucose metabolism. Journal of Clinical Endocrinology \& Metabolism, v. 72, p. 277282, 1991.

ANDERSON, T.; SCHEIN, M.; MCMENAMIN, M.G.; COONEY, D.A. Streptozotocin diabetes. Correlation with extent of depression of pancreatic islet nicotinamide adenine dinucleotide. The Journal of Clinical Investigation, v. 54, p. 672-677, 1974.

ANNIS, A.M.; CAULDER, M.S.; COOK, M.L.; DUQUETTE, D. Family History, Diabetes, and Other Demographic and Risk Factors Among Participants of the National Health and Nutrition Examination Survey 1999-2002. Preventing Chronic Disease, v. 2, n. 2, p.1-12, 2005.

ARSLANIAN, S.A.; KALHAN, S.C. Correlations between fatty acid and glucose metabolism. Potential explanation of insulin resistance of puberty. Diabetes, v. 43, n. 7, p. 908-14, 1994.

De acordo com:

ASSOCIAÇÃO BRASILEIRA DE NORMAIS TÉCNICAS. NBR 6023: Informação e documentação:: referências: elaboração. Rio de Janeiro, 2002. 
ARSLANIAN, S.A.;SUPRASONGSIN, C. Testosterone Treatment in Adolescents with Delayed Puberty: Changes in Body Composition, Protein, Fat and Glucose Metabolism. Journal of Clinical Endocrinology and Metabolism, v. 82, n. 10, p. 3213-3220, 1997.

ATKINSON, M.A.; EISENBARTH, G.S. Type 1 diabetes: new perspectives on disease pathogenesis and treatment. The Lancet, v. 358, p. 221-229, 2001.

BACCETTI, B.; MARCA, A.; PIOMBONI, P.; CAPITANI, S.; BRUNI, E.; PETRAGLIA, F.; LEO, V. Insulin-dependent diabetes in men is associated with hypothalamo-pituitary derangement and with impairment in sêmen quality. Human Reproduction, v. 17, n. 10, p.: 2673-2677, 2002.

BALLESTER,J.; MUÑOZ, M.C.; DOMÍNGUEZ, J.; RIGAU, T.;GUINOVART, J.J.;RODRÍGUEZ-GIL, J.E. Insulin-Dependent diabetes affects testicular function by FSH- and LH-linked mechanisms.Journal of Andrology, v. 25, n. 5, p. 706-18, 2004.

BEGUM, N.; DRAZNIN,B. Effect of streptozotocin-induced diabetes on GLUT-4 phosphorylation in rat adipocytes. The Journal of Clinical Investigation, v.90, p. 1254-1262, 1992.

BLONDEL, O.; BAILBE, D.; PORTHA, B. Insulin resistance in rats with non-insulindependent diabetes induced by neonatal n. 5 days) streptozotocin: evidence for reversal following phlorizin treatment. Metabolism, v. 39, n. 8, p. 787-93, 1990.

BONNER-WEIR, S.; TRENT, F.; HONEY, R.N.; WEIR, G.C. Responses of neonatal rat islet to streptozotocin. Diabetes, v. 30, p. 64-69, 1981.

BOUJON, C.E.; BESTETTI, G.E.; ABRAMO, F.; LOCATELLI, V.; ROSSI, G.L. The reduction of circulating growth hormone and prolactin in streptozotocin-induced diabetic male rats is possibly caused by hypothalamic rather than pituitary changes. Journal of Endocrinology, v. 145 , p. 19-26, 1995.

BRITO, L.C. A pinealectomia no período de desmame altera o metabolismo do tecido adiposo branco e retarda a puberdade em ratas. 2005. 104f. Tese $n$. Doutorado em Fisiologia) - Instituto de Ciências Biomédicas, Universidade de São Paulo, São Paulo, 2005.

BUCHOLTZ, D.C. et al. Regulation of pulsatile luteinizing hormone secretion by insulin in the diabetic male lamb. Biology of reproduction, v. 62, p. 1248-1255, 2000.

CALLAHAN, S.T.; MANSFIELD, M.J. Type 2 diabetes mellitus in adolescents. Current Opinion in Pediatrics, v. 12, n. 4, p.310-5, 2000. 
CASABIELL, X.; PIÑEIRO, V.; VEGA, F.; CRUZ, L.F.D., DIÉGUEZ, C.;CASANUEVA, F.F. Leptin, Reproduction and Sex Steroids. Pituitary, v. 4, p. 93-99, 2001.

CLÉMENT K.; VAISSE C.; LAHLOU N. A mutation in the human leptin receptor gene causes obesity and pituitary dysfunction. Nature, v. 392, p. 398-401, 1998.

COHEN, B.; NOVICK, D.; RUBINSTEIN, M. Modulation of insulin activities by leptin. Science, v. 274, p. 1185-1188, 1996.

CONNOR, P.J.; GREGG, E.; RUSB, W.A.; CHERNEY, L.M.; STIFFMAN, M.N; ENGELGAU, M.M. Diabetes: How Are We Diagnosing and Initially Managing It? Annals of Family Medicine, v. 4, n. 1, p. 15-22, 2006.

CONSTANZO, L.S. Fisiologia Reprodutora. In. SILVA MOREIRA, A.J.M.; SALLES, A.D.; CAMPOS, J.P. (Trad.). Fisiologia. Rio de Janeiro: Elsevier, 2004. p. 423-427.

DIGIROLAMO, M.; MEDLINGER, S.; FERTIG, W.A. A simple method to determine fat cell size and number in four mammalian species. American Journal of Physiology, $v$. 221, n. 3, p. 850-888, 1971.

DINULOVIC, D.; RADONJIC, G. Diabetes mellitus/male infertility. Archives of Andrology, v. 25, p. 277-293, 1990.

DOLE, V.P.; MEINERTZ, H. Microdetermination of long-chain fatty-acids in plasma and tissues. The Journal of Biological Chemistry, v.235, n. 9, p. 2595-2599, 1960.

DUTLOW, C.M.; RACHMAN, J.; JACOBS, T.W.; MILLAR, R.P. Prepubertal increases in gonadotropin-releasing hormone mRNA, gonadotropin-releasing hormone precursor, and subsequent maturation of precursor processing in male rats. Journal of Clinical Investigation, v. 90, n. 6, p. 2496-2501, 1992.

EURODIAB ACE Study Group. Variation and trends in incidence of childhood-onset diabetes in Europe. Lancet, v. 355, p. 873-876, 2000.

FOSTER, D. L.; OLSTER D. M.; YELLON S. Introduction. In: VENTUROLI, S.; FLAMIGNI, C.; GIVENS, J.R. (Ed.). Adolescence in Females. Chicago: Year Book Medical Publishers, 1985.

GENUTH, S.M. Hormônios das ilhotas pancreáticas. In: BERNE, R.M. et al. Fisiologia. 5a ed. Rio de Janeiro: Elsevier, 2004. p.815-843.

GENUTH, S.M.; As glândulas reprodutoras. In: BERNE, R.M. et al. Fisiologia. 5a ed. Rio de Janeiro: Elsevier, 2004. p. 981-1042. 
GIORGINO F.; CHEN J.; SMITH R.J. Changes in tyrosine phosphorylation of insulin receptors and a 170,000 molecular weight nonreceptor protein in vivo in skeletal muscle of streptozotocin-induced diabetic rats: effects of insulin and glucose. Endocrinology, v. 130, p.1433-1444, 1992.

GORAN, M.; GOWER, B. Longitudinal Study on Pubertal Insulin Resistance. Diabetes, v. 50 , p. $2444-2450,2001$.

GROMOLL, J.; SIMONI, M.; NORDHOFF, V.; BEHRE, H.M.; GEYTER, C.D.; NIESCHLAG, E. Functional and clinical consequences of mutations in the FSH receptor. Molecular and Cellular Endocrinology, 125:177-182, 1996.

GUEORGUIEV, M.; GOTH, M.I.; KORBONITS, M. Leptin and Puberty: A review. Pituitary, v. 4, p. 79-86, 2001.

GUYTON, A.C. The pituitary hormones and their control by the hypothalamus. In: TEXTBOOK of Medical Physiology. Philadelphia: W.B. Saunders Company, 1991. p. 822-27.

HAYNES, A.; BOWER, C.; BULSARA, M.K.; JONES, T.W.; DAVIS, E.A. Continued increases in the incidence of childhood type 1 diabetes in population-based Australian sample n. 1985-2002). Diabetologia, v. 47, p. 866-870, 2004.

HONNOR, R.C.; DHILLON, G.S.; LONDOS, C. CAMP-dependent protein kinase and lipolysis in rat adipocytes. Cell preparation, manipulation, and predictability in behavior. Journal of Biological Chemistry, v.200, n. 15, p. 122-129, 1985.

JOHNSON, L.M.; SIDMAN, R.L. A reproductive endocrine profile in the diabetes (db) mutant mouse. Biology of Reproduction, v. 20, p. 552-559, 1979.

JOUBERT, Y.; TOBIN, C.; LEBART, M.C.Testosterone-induced masculinization of the rat levator any muscle during puberty. Developmental Biology, v. 162, p. 104-110, 1994.

JOVANOVIC, L.; PETTITT, D.J. Gestational Diabetes Mellitus. The Journal of The American Medical Association, v. 286, n. 20, p. 2516-2518, 2001.

JUNOD, A.; LAMBERT, A.; STAUFFACHER, W.; RENOLD, A. Diabetogenic Action of Streptozotocin: Relationship of Dose to Metabolic Response. The Journal of Clinical Investigation, v.48, p. 2129-2139, 1969.

KAHN, B.B.; FLIER, J.S. Obesity and Insulin resistance. The Journal of Clinical Investigation, v. 106, n. 4, p. 473-481, 2000. 
KARNIELI, E.; HISSIN, P.J.; SIMPSON, I.A.; SALANS, L.B.; CUSHMAN, S.W. The Journal of Clinical Investigation, v. 68, p. 811-814, 1981.

KERGOAT, M.; PORTHA, B. In vivo hepatic and peripheral insulin sensitivity in rats with non-insulin-dependent diabetes induced by streptozotocin.Assessment with the insulin-glucose clamp technique. Diabetes, v. 34, n. 11, p. 1120-6, 1985.

KETELSLEGERS, J. M.; HETZEL, W. D.; SHERINS R, J.;CATT K. J. Developmental changes in testicular gonadotropin receptors: plasma gonadotropins and plasma testosterone in the rat. Endocrinology, v. 103, p. 212-222, 1978.

KIM, E.; SOHN, S.; LEE, M.; JUNG, J.; KINEMAN, R.D.; PARK, S. Differential responses of the growth hormone axis in two rat models of streptozotocin-induced insulinopenic diabetes. Journal of Edocrinology, v.188, p. 263-270, 2006.

KOBAYASHI, M.; OLEFSKY, J.M. Effects of streptozotocin-induced diabetes on insulin binding, glucose transport, and intracellular glucose metabolism in isolated rat adipocytes. Diabetes, v. 28, p. 87-95, 1979.

KORENBROT, C.C.; HUHTANIEMI, I.T.; WEINER, R.I. Preputial separation as an external sign of pubertal development in the male rat. Biology of Reproduction, v. 17, p. 298-303, 1977.

LIMA, F.B.; MATSUSHITA, D.H.; HELL, N.S.; DOLNIKOFF, M.S.; OKAMOTO, M.M.; CIPOLLA-NETO, J. The regulation of insulin action in isolated adipocytes. Role of periodicity of food intake, time of the day and melatonin. Brazilian Journal of Medical Research, v. 27, p. 995-1000, 1994.

LIVINGSTONE, C.; COLLISON, M. Sex steroids and insulin resistance. Clinical Science, v. 102, p. 151-166, 2002.

LOTT, J. A.; TURNER, K. Evaluation of Trinder's glucose oxidase method for measuring glucose in serum and urine. Clinical Chemistry, v. 21, p. 1754-1760, 1975.

MAEDLER, K.; SERGEEV, P.; EHSES, J.A.; MATHE, Z.; BOSCO, D.; BERNEY, T.; DAYER, J.; REINECKE, M.; HALBAN, P.A., DONATH, M.Y. Leptin modulates $\beta$ cell expression of IL-1 receptor antagonist and release of IL-1 $\beta$ in human islet. PNAS, $v$. 101, n. 21, p. 8138-8143, 2004.

MANTZOROS, C.S.; FLIER, J.S.; ROGOL, A.D. A Longitudinal Assessment of Hormonal and Physical Alterations during Normal Puberty in Boys. V. Rising Leptin Levels May Signal the Onset of Puberty. Journal of Clinical Endocrinology and Metabolism, v. 82, n. 4, p. 1066-1070, 1997. 
MASHARANI, U.; KARAM, J.H.; GERMAN, M.S. Hormônios pancreáticos e diabetes melito. In: CONSENDEY, C.H.; FIGUEIREDO, J.E.; VOEUX, P.L.; JACOBSON, R.; SETÚBAL, S. (Trad.). Endocrinologia Básica e Clínica. Rio de Janeiro: McGraw-Hill, 2006. p. 557-560.

MAURAS, N.; BLIZZARD, R.M.; LINK, K.; JOHNSON, M.L.; ROGOL, A.D.; VELDHUIS, J.D. Augmentation of growth hormone secretion during puberty: evidence for a pulse amplitude-modulated phenomenon. Journal of Clinical Endocrinology \& Metabolism, v. 64, p. 596-601, 1987.

MONOSSON, E.; KELCE, W.R.; LAMBRIGHT, C.; OSTBY, J.;GRAY, L.E. Peripubertal exposure to the antiandrogenic fungicide, vinclozolin, delays puberty, inhibits the development of androgen-dependent tissues, and alters androgen receptor function in the male rat. Toxicology and Industrial Health, v. 15, p. 65-79, 1999.

MOKDAD, A.H. et al. The continuing epidemics of obesity and diabetes in the United States. JAMA, v. 286, p. 1195-1220, 2001.

MORAN, A.; JACOBS, D.R.; STEINBERGER, J.; HONG,C.; PRINEAS,R.; LUEPKER,R.; SINAIKO,A.R. Insulin resistance during puberty. Diabetes, v. 48, p. 2039-2044, 1999.

MORAN, A.; JACOBS, D.; STEINBERGER, J.; COHEN, P.; HONG,C.P.; PRINEAS, R.; SINAIKO, A. Association between the Insulin Resistance of Puberty and the InsulinLike Growth Factor-I/Growth Hormone Axis. The Journal of Clinical Endocrinology \& Metabolism, v. 87, n. 10, p.4817-4820, 2002.

MURAO, S. et al. Supression of episodic growth hormone secretion in streptozotocininduced diabetic mice: time-course studies on the hypothalamic pituitary axis. Endocrinology, v. 136, n. 10, p. 4498-4504, 1995.

OGLESBY,A.K.; SECNIK, K. ;BARRON,J.; AL-ZAKWANI, I.; LAGE, M.J. The association between diabetes related medical costs and glycemic control: $A$ retrospective analysis. Cost Effectiveness and Resource Allocation, v. 4, n. 1, p. 18, 2006.

OJEDA, S. R.; WHITE, s.s.; URBANSKI, H.F.; AGUADO L. I. The onset of female puberty: underlying neuroendocrine mechanisms. Neuroendocrinology Perspectives, v. 3, p. 225, 1984.

OJEDA, S.R.; LOMNICZIA, A.; MASTRONARDI, C.; HEGER, S.; ROTH, C.; PARENT, A.S.; MATAGNE, V.; MUNGENAST, A.E. The neuroendocrine regulation of puberty: is the time ripe for a systems biology approach? Endocrinology, v. 147, n. 3, p. 11661174, 2006. 
OKSANEN, A. Testicular lesions of streptozotocin diabetic rats. Hormone Research, v. 6 , p. 138-44, 1975.

OLCHOVSKY, D. et al. Altered Pituitary Growth Hormone $(\mathrm{GH})$ regulation in streptozotocin-diabetic rats: a combined defect of hypothalamic somatostatin and $\mathrm{GH}$ releasing factor. Endocrinology. v. 126, n. 1, p. 53-61, 1990.

ONKAMO, P.; VÄÄNÄNEN, S.; KARVONEN, M.; TUOMILEHTO, J. Wordwild increase in incidence of type 1 diabetes - the analysis of the data on published incidence trends. Diabetologia, v. 42, p. 1395-1403, 1999.

PAIK, S.; FLEISCHER, N.; SHIN, S. Insulin-dependent diabetes mellitus induced by subdiabetogenic doses of streptozotocin: Obligatory role of cell-mediated autoimmune processes. Proceedings of National Academy of Sciences, v. 77, n. 10, p. 61296133, 1980.

PAYNE, A. H.; KELCH, R. P.; MURONO E. P. Hypothalamic, pituitary and testicular function during sexual maturation of the male rat. Journal of Endocrinology, v. 72, p. 17-26, 1977.

PI-SUNYER, F.X. Medical Hazards of Obesity. Annals of Internal Medicine, v. 119, n. 7, p. 655-660, 1993.

PORTHA, B.; BLONDEL, O.; SERRADAS, P.;EVOY, R.M.;GIROIX, M.H.; KERGOAT, M.; BAILBE, D. The rat models of non-insulin dependent diabetes induced by neonatal streptozotocin. Diabete \& Metabolisme, v. 15, n. 2, p. 61-75, 1989.

POLDERMAN, K.H.; GOOREN, L.J.; ASSCHEMAN, H., BAKKER, A.; HEINE, R.J. Induction of Insulin Resistance by Androgens and Estrogens. Journal of Clinical Endocrinology and Metabolism, v. 79, n. 1, p. 265-271, 1994.

REHMAN, K.; BESHAY, E.; CARRIER, S. Diabetes and male sexual function. Sez. Reprod. Med., v. 1, n. 1, p. 29-33, 2001.

RODBELL M. Metabolism of isolated fat cells. Effects of hormones on glucose metabolism and lipids. Journal of Biological Chemistry, v. 239, p. 357-380, 1964.

RODRIGUES, T.M.B.; SILVA, I.N. Estatura final dos pacientes com diabetes mellitus tipo 1. Arquivos Brasileiros de Endocrinologia e Metabologia, v. 45, n. 1, p. 108$114,2001$.

RODRIGUEZ-RIGAU, L.J. Diabetes and male reproductive function. Journal of Andrology, v. 1, n. 3, p. 105-110, 1980. 
ROSENBLOOM, A.L.; JOE, J.R.; YOUNG, R.S.; WINTER, W.E. Emerging Epidemic of Type 2 Diabetes in Youth. Diabetes Care, v. 22, p. 345-354, 1999.

ROSSINI, A.; LIKE, A.; CHICK, W.; APPEL, M. Studies of streptozotocin- induced insulitis and diabetes. Proceeding of National Academy of Sciences, v. 74, n. 6, p. 2485-2489, 1977.

SAAD, R.J.; KEENAN, B.S.; DANADIAN, K.; LEWY, V.D.; ARSLANIAN, S.A. Dihydrotestosterone Treatment in Adolescents with Delayed Puberty: Does it Explain Insulin Resistance. The Journal of Clinical Endocrinology \& Metabolism, v. 86, n. 10, p. 4881-4886 2001.

SAAD, M.J.A.; ARAKI, E.; MIRALPEIX, M.; ROTHENBERG, P.L.; WHITE, M.F.; KAHN, R. Regulation of Insulin Receptor Substrate-1 in Liver and Muscle of Animal Models of Insulin Resistance. Journal of Clinical Investigation, v. 90, p. 1839-1849, 1992.

SEETHALAKSHMI, L.; MENON, M.; DIAMOND, D. The effect of streptozotocininduced diabetes on the neuroendocrine-male reproductive tract axis of the adult rat. Journal of Urology, v. 138, p. 190-194, 1987. Abstract

SERRADAS, P.; BLONDEL, O.; BAILBE, D.; PORTHA, B. Bonfluorex normalizes hyperglycemia and reverses hepatic insulin resistance in STZ-induced diabetic rats. Diabetes, v. 42, n. 4, p. 564-70, 1993.

SEXTON, W.J.; JAROW, J.P. Effect of diabetes mellitus upon male reproductive function. Urology, v. 49, n. 4, p. 508-513, 1997.

SJÖBERG, P.; LINDQVIST, N.G.; PLÖEN, L. Age-dependent response of the rat testes to di(2 ethylhexyl) phthalate. Environmental Health Perspectives, v. 65, p. 237-242, 1986.

SKYLER, J.S. et alii. Effects of insulin in relatives of patients with type 1 diabetes mellitus. The New England journal of medicine, v. 346, n. 22, p. 1685- 1691, 2002.

STEGER, R.W.; RABE, M.B. The effect of diabetes mellitus on endocrine and reproductive function. The Society for Experimental Biology and Medicine. v. 214, p. 1-11, 1997.

STROBEL A.; ISSAD T.; CAMOIN L.; OZATA M.; STROSBERG A.D. A leptin missense mutation associated with hypogonadism and morbid obesity. Nature Genetics, v. 18, p. 213-215, 1998. 
SUDHA, S.; VALLI, G.; MARY JULIE, P.; ARUNAKARAN, J.; GOVINDARAJULU, P.; BALASUBRAMANIAN, K. Influence of streptozotocin-induced diabetes and insulin treatment on the pituitary-testicular axis during sexual maturation in rats. Exp. Clin. Endocrinol. Diabetes. v. 107, p.14-20, 1999.

TAKADA, J. Diabetes Mellitus induzido no período neonatal: caracterização do metabolismo celular. 2003. 98 p. Dissertação (Mestrado em Fisiologia) - Instituto de Ciências Biomédicas, Universidade de São Paulo, São Paulo, 2003.

REPORT OF THE EXPERT COMMITTEE ON THE DIAGNOSIS AND CLASSIFICATION OF DIABETES MELLITUS. Diabetes Care, v. 26, n. 1, p. 5-20, 2003.

THORNER, M.O.; VANCE, M.L. Growth Hormone. Journal of Clinical Investigation, v. 82 , p. $745-47,1988$.

TRAVERS, S.H.; JEFFERS, B.W.; BLOCH, C.A.; HILL, J.O.; ECKEL, R.H. Gender and Tanner stage differences in body composition and insulin sensitivity in early pubertal children. Journal of Clinical Endocrinology \& Metabolism, v. 80, p. 172-178, 1995.

UYSAL, K.T.; WIESBROCK, S.M.; MARINO, M.W.; HOTAMISLIGIL, G.S. Protection fromobesityinduced insulin resistance in mice lacking TNF-a function. Nature, v. 389, p. 610-614, 1997.

WATANOBE, H.; SCHIOTH, H.B. Postnatal profile of plasma leptin concentrations in male and female rats: Relation with the maturation of the pituitary-gonadal axis. Regulatory Peptides, v. 105, p. 23-28, 2002.

WEIR, G.C. et al. Islet secretion in a new experimental model for non-insulin dependent diabetes. Diabetes, v. 30, p. 590-95, 1981.

WEIR, G.C. et al. B- Cell adaptation and decompensation during the progression of diabetes. Diabetes, v. 50, n. 1, p. 154-159, 2001.

WOOD, D.W. Hormônios. In: SOARES VEIGA, M.F. (Trad.). Princípios de Fisiologia Animal. São Paulo: Editora Polígono, 1973. p.318-319.

ZIMMET, P.; ALBERTI, M.M.; SHAW, J. Global and societal implications of the diabetes epidemic. Nature, v. 414, p. 782-787, 2001.

ZANATO, V.F.; MARTINS, M.P.; ANSELMO-FRANCI, J.A.; PETENUSCI, S.O.; LAMANO-CARVALHO, T.L. Sexual Development of male wistar rats. Brazilian Journal Medical and Biological Research, v. 27, p. 1273-1280, 1994. 